OMAE2014-23864

\title{
A MULTI-BODY ALGORITHM FOR WAVE ENERGY CONVERTERS EMPLOYING NONLINEAR JOINT REPRESENTATION
}

\author{
Davide Padeletti* \\ Centre for Ocean Energy Research \\ NUI Maynooth \\ Maynooth, Co. Kildare, Ireland \\ Email: dpadeletti@eeng.nuim.ie
}

\author{
Ronan Costello \\ Centre for Ocean Energy Research \\ NUI Maynooth \\ Maynooth, Co. Kildare, Ireland \\ Email: rcostello@eeng.nuim.ie
}

\author{
John V. Ringwood \\ Centre for Ocean Energy Research \\ NUI Maynooth \\ Maynooth, Co. Kildare, Ireland \\ Email: john.ringwood@eeng.nuim.ie
}

\begin{abstract}
When large relative displacements take place between the bodies in a multi-body Wave Energy Conversion system linearisation of the constraints on motion imposed by the joints between the bodies is no longer valid and a non-linear timedomain analysis is necessary. As a part of the Techno-Economic Optimisation of Wave Energy Conversion (TEOWEC) software, which has been developed at the Centre for Ocean Energy Research (COER), NUI Maynooth, we developed an algorithm for the dynamic simulation of Multi-Body Systems for Wave Energy Conversion (MBS4WEC) with fully non-linear representation of the body-to-body joints. The algorithm is based on the Jointcoordinate formulation, which provides a systematic procedure to transform the mixed differential-algebraic equations of motion in body coordinates, for open chain systems, to a minimal set of ODEs. When a closed-loop chain occurs, the same method can be adopted by removing one or more kinematic joints from each loop. Knowing the topology of the system, a path matrix is generated and together with the formulation of data structures representing the body-to-body joints, the Velocity Transformation Matrix is computed. The main advantage of this approach is a fast and automatic generation of the Velocity Transformation Matrix, which leads to a higher computational efficiency, especially for complex systems. This paper presents the equations underpinning the method together with results for simulation of two specimen floating multi-body systems. These two are a simple multi-body hinged barge and a device with a sliding internal
\end{abstract}

\footnotetext{
*Address all correspondence to this author.
}

reaction mass. In each case the results are contrasted to the results produced by a linearised analysis of the same system.

\section{NOMENCLATURE}

P Velocity transformation matrix

$\Pi$ Path matrix

$\Phi$ Jacobian matrix of constraint equations

$v$ Generalised velocity vector

$\eta \quad$ Generalised position vector

M Generalised mass matrix

G Hydrostatic restoring matrix

D Radiation dumping matrix

s Independent velocity vector

q Independent position vector

$\lambda$ Lagrangian multiplier vector

$\tau$ Generalised force vector

$\tau_{E} \quad$ Wave-excitation force vector

$\tau_{H} \quad$ Hydrostatic force vector

$\tau_{\text {rad }}$ Radiation force vector

$\tau_{S D A} \quad$ Spring-damping-actuator force vector

$\tau_{\text {moor }}$ Mooring force vector

\section{INTRODUCTION}

A multi-body system is a collection of at least two bodies connected to each other by joints and acted upon by forces and 
torques. Multi-body dynamics analysis has been studied in many scientific fields : robotics, mechanical and space engineering, computer science, biomedical engineering, etc. The equations underpinning the motion are the Newton-Euler constrained equations [1], which represent a mixed set of Differential-Algebraic Equation (DAE). A minimum number of variables can be selected, reducing the dynamics of the system to a minimal set of Ordinary Differential Equations (ODEs). Three different formulations are normally adopted to formulate the equations of motion : the body-coordinate (or absolute coordinate), the pointcoordinate and the joint-coordinate method [2]. In this paper we made use of the joint-coordinate approach for open-chain systems. In the next sections we will give an overview of the mathematical model, then we will explain the implementation of the MBS4WEC tool and finally we will discuss two simple test case applications : a 3 hinged barges system and a floating body with internal sliding reaction mass device. The results are then compared to the linearised model available in TEOWEC.

\section{MATHEMATICAL FORMULATION}

Nikravesh [3] represents the Newton-Euler equations of motion for a constrained system of rigid bodies as

$$
\mathbf{M} \dot{v}+\Phi^{T} \lambda=\tau
$$

where $\mathbf{M}$ is the generalised mass matrix, $\dot{v}$ the time derivative of the generalised velocities, $\lambda$ is an array of Lagrange multipliers containing as many elements as the number of constraints describing the joint and $\Phi$ is the Jacobian matrix of the constraint equation. Velocity and acceleration constraints are expressed as

$$
\begin{gathered}
\dot{\Phi} v=0 \\
\Phi \dot{v}+\dot{\Phi} v=0
\end{gathered}
$$

The vector $\tau$ represents the generalized forces and it can be decomposed as

$$
\tau=\tau_{E}+\tau_{H}+\tau_{\text {rad }}+\tau_{S D A}+\tau_{o t h e r}
$$

where $\tau_{E}, \tau_{H}$ and $\tau_{\text {rad }}$ represent the excitation, hydrostatic restoring and radiation damping forces respectively. $\tau_{S D A}$ is the force vector due to spring-damper-actuator contribution. $\tau_{\text {other }}$
TABLE 1. MAIN FORCES COMPONENTS.

\begin{tabular}{c}
\hline Reduced Forces \\
\hline$\tilde{\tau}_{E}=\mathbf{P} \tau_{E}$ \\
$\tilde{\tau}_{H}=-\left(\mathbf{P G P} \mathbf{P}^{T}\right) \mathbf{q}$ \\
$\tilde{\tau}_{r a d}=-\left(\mathbf{P D P} \mathbf{P}^{T}\right) \mathbf{s}$ \\
$\tilde{\tau}_{S D A}=\mathbf{P} \tau_{S D A}$ \\
\hline
\end{tabular}

includes other external forces, such as moorings, power take-off or viscous forces.

When trying to reduce the number of equations which solve the Newton-Euler motion problem for a constrained multi-body system, a minimal set of coordinates has to be detected. And this will depend on the kinematics of the system. If we apply a velocity transformation, we can then define the generalised velocities $v$ in terms of the independent velocities $\mathbf{s}$.

$$
v=\mathbf{P}^{T} \mathbf{s}
$$

Deriving the (5) we obtain

$$
\dot{v}=\mathbf{P}^{T} \dot{\mathbf{s}}+\dot{\mathbf{P}}^{T} \mathbf{S}
$$

$\mathbf{P}$ is called the velocity transformation matrix and $\dot{\mathbf{P}}$ is its time derivative. Matrix $\mathbf{P}^{T}$ is orthogonal to the system Jacobian:

$$
\Phi \mathbf{P}^{T}=0
$$

Substituing Eqns. (4-6) in Eqn. (1), then considering (7) and the relation between the reduced and generalised forces as given in Tab. 1, we lead at the following formulation

$$
\left(\mathbf{P M P} \mathbf{P}^{T}\right) \dot{\mathbf{s}}+\left(\mathbf{P D P}^{T}+\mathbf{P M} \dot{\mathbf{P}}^{T}\right) \mathbf{s}+\left(\mathbf{P G P}^{T}\right) \mathbf{q}=\mathbf{P}\left(\tau_{E}+\tau_{S D A}\right)
$$

Equations (8) describe a system of differential equations with the same number of equations as the number of degrees of freedom (DOFs) of the system. In order to compute the matrices $\mathbf{P}$ and $\dot{\mathbf{P}}$ we use the joint-coordinate approach as in [4]. The method is based on representing a set of joint coordinates (or velocities) as the independent coordinates and then transforming 
the equations of motion to a minimal set of second order ODEs. The independent variables are mostly relative coordinates associated with the kinematic joints that connect the bodies forming a multi-body system, generally called an open-chain system. The open-chain system is like a tree. The first step it to select a base body, which is called root. Then we move to an adjacent body via its joint, then to another body and so on until no further bodies can be reached. The last element is called leaf and the ordered sequence of bodies is a branch. When following this scheme, bodies are numbered in an ascending order, so that the topology of the system can be reconstruct. Tracking the tree topology is crucial to correctly build the $\mathbf{P}$ matrix. This is done by identifying a path matrix as defined by Jerkovsky [5]

$$
\Pi_{i j}= \begin{cases}1, & \text { if body } j \text { is between the root and the } i \text { body } \\ 0, & \text { otherwise }\end{cases}
$$

In the following section we will explain how this mathematical formulation has been implemented in MBS4WEC.

\section{THE MBS4WEC IMPLEMENTATION}

As a part of the TEOWEC software, the MBS4WEC tool requires that some inputs are previously generated from it, as shown in Fig. 1. probDat and hydroDat are input data structures representing the hydrodynamic problem for the WAMIT BEM solver. All the hydrodinamic parameters for a selected WEC concept are at this stage computed, and the hydrodynamic forces are decomposed into excitation, radiation damping and restoring components.

The jointData is a data structure introduced to keep track of all the kinematic constraints of the multi-body chain. At this preliminary stage, this input data is manually inserted by the user via command-line interface. Any kind of basic joint can be here chosen.

The aim of the preProcessing block is to solve the topological sorting problem, using the Depth-First Search (DFS) algorithm [6]. Here system elements are uniquely named and the paths, i.e. the consecutive sequences of bodies going from the root to the leaves, evaluated.

In the simulation function all the inputs together with the initial conditions are collected to solve the set of ODEs. The solver implemented is the Runge-Kutta 4th/5th-order method.

Finally postProcessing rearranges the solution variables and plots position and attitude of each body forming the system.

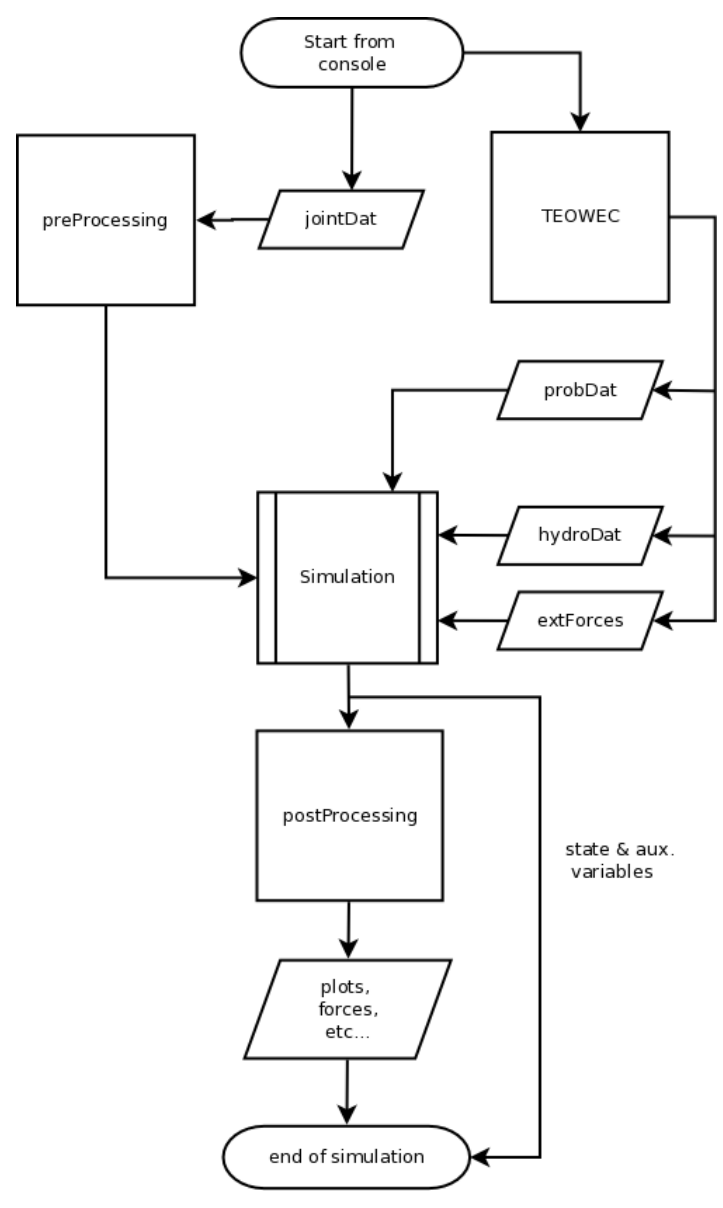

FIGURE 1. MBS4WEC FLOWCHART.

\section{RESULTS \\ Three hinged barges}

Figure 2 shows the three hinged barge setup. The first body on the left is the root and the one on the very right is the only leaf of this short 3-body open-chain. The body-fixed frames $b_{i}=\left\{O_{i} x_{i} y_{i} z_{i}\right\}$ have their origins in the center of mass of each body. The global frame (where all the equations are reduced) has at initial time same origin as the $b_{2}$ coordinate frame. The independent velocites $\mathbf{s}$ were chosen to be the 6 generalised velocities (traslational and angular velocity) of the first body, plus the relative angular displacement rates between body1-body $2\left(\dot{\alpha}_{2}\right)$ and body2-body3 $\left(\dot{\alpha}_{3}\right)$

$$
\mathbf{s}=\left[\begin{array}{llllllll}
v_{x_{1}} & v_{y_{1}} & v_{z_{1}} & \omega_{x_{1}} & \omega_{y_{1}} & \omega_{z_{1}} & \dot{\alpha}_{2} & \dot{\alpha}_{3}
\end{array}\right]
$$

Two revolute joints are located at point $P_{2}$ and $P_{3}$. For both, the revolute axis is parallel to the global $y$-axis, laying in the plane of the undisturbed water free surface at rest. 


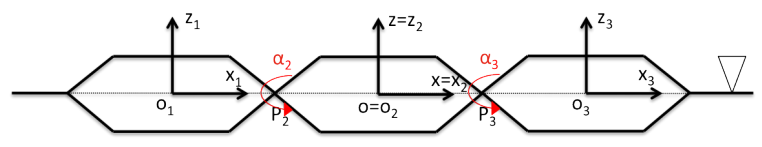

FIGURE 2. HINGED BARGES SETUP.
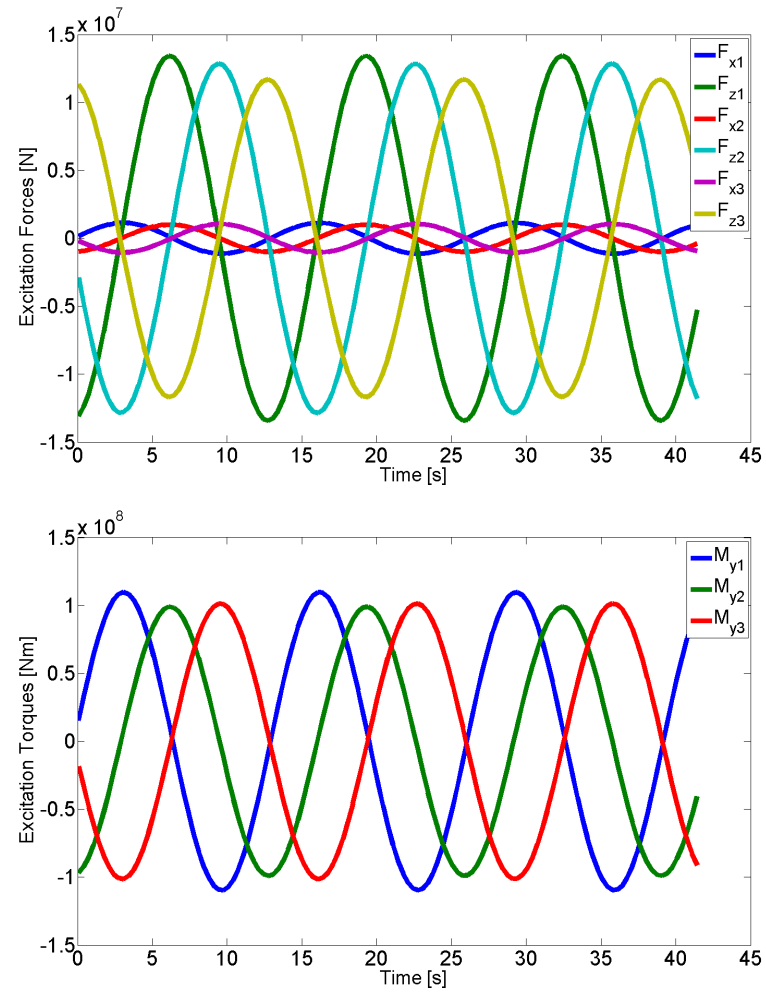

FIGURE 3. EXCITATION FORCES AND TORQUES.

We modelled some mooring loads acting on the root only, as linear spring and damper

$$
\tau_{\text {moor }}=-\mathbf{B}_{\text {moor }} v-\mathbf{G}_{\text {moor }} \eta
$$

Finally, we ran a test assuming monochromatic, unidirectional waves with period $f=6.8966 \mathrm{~s}$ and wave height $H=6 \mathrm{~m}$. Since the waves front is parallel to the longitudinal axis of the barges, in the linear analysis the significant modes are represented surge, heave and pitch. Furthermore, the calculation of the $\mathbf{P}$ matrix turns out to be more straightforward (see [7]). Figure 3 shows the excitation force and torque components acting on the three bodies as calculated by WAMIT.

As depicted in Fig. 4, results for surge, heave and pitch from the linear and non-linear analysis coincide after few seconds of
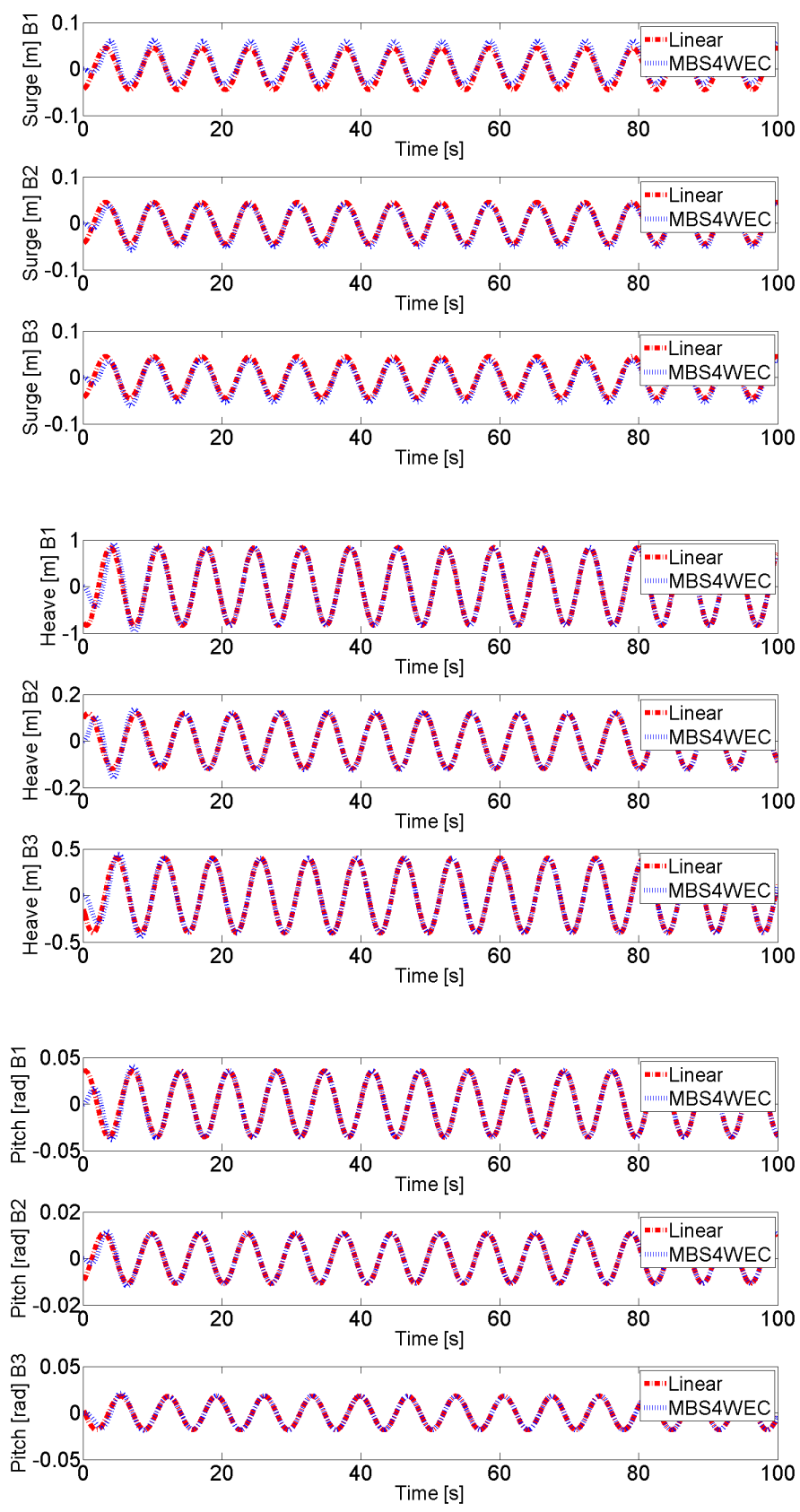

FIGURE 4. SURGE, HEAVE AND PITCH.

simulation, showing that there is no particular suitability in chosing the MBS4WEC method for this particular test case. 


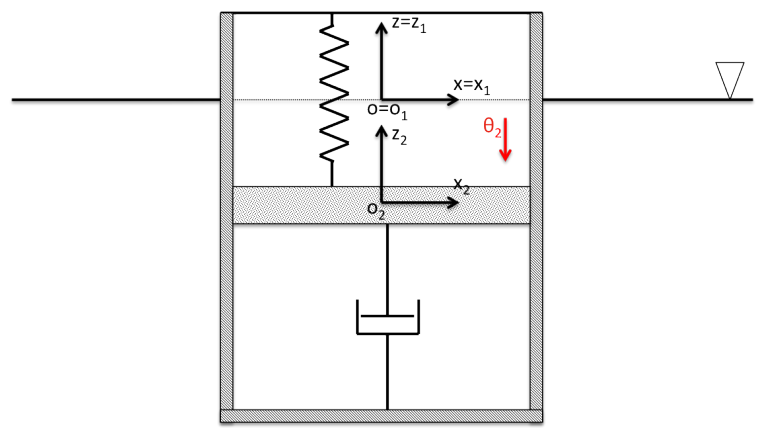

FIGURE 5. INTERNAL REACTION MASS.

\section{The sliding internal reaction mass}

The second test case is depicted in Fig. 5. The model is formed by a heaving, vertically axi-symmetric buoy with an internal reaction mass. The body fixed frame $b_{1}$ has its origin in the waterline as well as the $x y$ plane, while the $z$-axis coinciding to the longitudinal axis. The second body frame $b_{2}$ is fixed to the reaction mass, having origin in its center of mass. The global frame is assumed to coincide with $b_{1}$ at initial time. The two bodies are connected by a translational joint, which allows only one relative DoF. So the overall system can be described by 7 velocity coordinates, the six generalised velocities for the buoy, and one relative translational velocity $\dot{\theta_{2}}$.

$$
\mathbf{s}=\left[\begin{array}{lllllll}
v_{x_{1}} & v_{y_{1}} & v_{z_{1}} & \omega_{x_{1}} & \omega_{y_{1}} & \omega_{z_{1}} & \dot{\theta}_{2}
\end{array}\right]
$$

The hydrostatic and hydrodynamic forces and torques act only on the buoy since it is the only "wet" element. The translational spring instead applies force and moment $\tau_{\text {spring }}$ on both bodies. Defining $\mathbf{u}_{s}$ the unit vector of the spring axis, and assuming that the undeformed length $l_{0}$ and spring stiffness $k$ are known, the resultant force on first body is expressed ase

$$
\mathbf{f}_{s}=k\left(l-l_{0}\right) \mathbf{u}_{s}
$$

The force acting on the reaction mass will have same absolute value but opposite direction. Moreover, these forces have moment arms, so that $\tau_{\text {spring }}^{i}$ can be written as

$$
\tau_{\text {spring }}^{i}=\left[\begin{array}{c}
\mathbf{f}_{s}^{i} \\
{\widetilde{\mathbf{s}_{s}^{i}}}_{\mathbf{f}_{s}^{i}}^{i}
\end{array}\right]
$$

$\widetilde{\mathbf{S}_{s}^{i}}$ representing the skew matrix of the vector between the point of application of the spring force and the center of mass of body $i$. The effect of the translational damper is similar to the spring, whereas here the time rate of change of the damper lenght $i$ must be computed. The damping force will be then equal to

$$
\mathbf{f}_{d}=b \dot{l} \mathbf{u}_{d}
$$

Similar to Eqn. 14 we can write the damper contribution as

$$
\tau_{\text {damper }}^{i}=\left[\begin{array}{c}
\mathbf{f}_{d}^{i} \\
{\widetilde{\mathbf{s}_{d}^{i}}}_{d_{d}^{i}}^{\mathbf{f}_{d}^{i}}
\end{array}\right]
$$

where $\widetilde{\mathbf{s}_{d}^{i}}$ represents the skew matrix of the vector between the point of application of the damping force and the center of mass of body $i . \tau_{S D A}$ results in the sum of Eqn. 14 and 16. Due to the presence of a "dry" body, the reaction mass, the contribution of the gravitational force has to be taken into account

$$
\tau_{\text {grav }}=\left[\begin{array}{llllll}
0 & 0 & -g m & 0 & 0 & 0
\end{array}\right]^{T}
$$

Also for this test we moored the floating buoy and assume the same wave condition as used for the three hinged barge test. A comparison for surge, heave and pitch for both methods is given in Fig. 6.

\section{CONCLUSION}

For both the hinged barge and the buoy with internal reaction mass the linear and MBS4WEC simulations gave very close results. This appears to be a good proof that the joint-coordinate method can be applied to multi-body WEC systems. However, because the rotations in these test cases are small the advantages of the non-linear representation of the joints are not demonstrated to best effect by this test.

\section{ACKNOWLEDGMENT}

The authors would like to thank Enterprise Ireland for financing the project.

\section{REFERENCES}

[1] Shabana, A., 2005. Dynamics of Multibody Systems. Cambridge University Press.

[2] de Jalón, J., and Bayo, E., 1994. Kinematic and dynamic simulation of multibody systems: the real-time challenge. Mechanical engineering series. Springer-Verlag. 

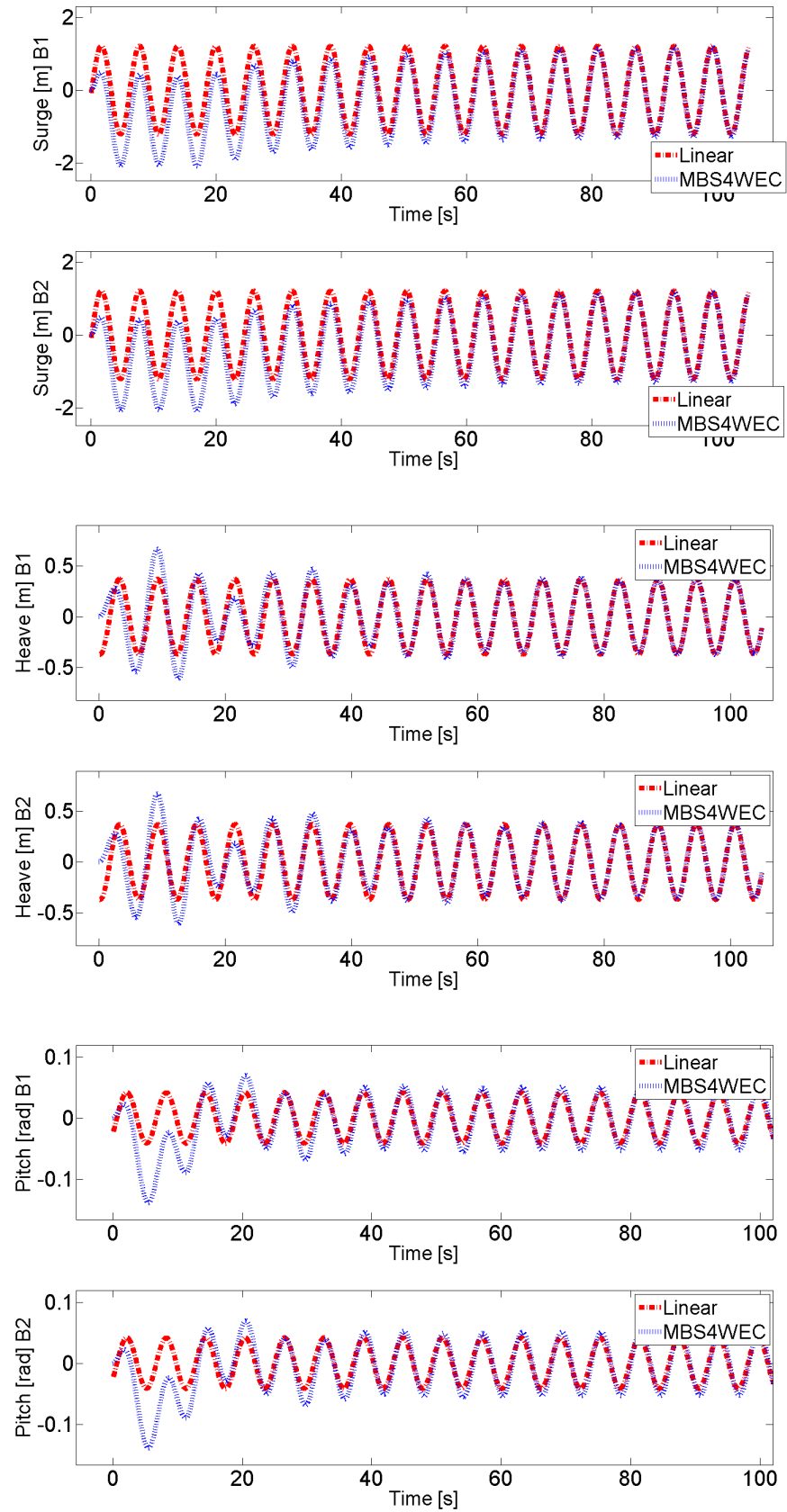

FIGURE 6. SURGE, HEAVE AND PITCH.

[3] Nikravesh, P. E., 1988. Computer-Aided Analysis of Mechanical Systems. Prentice Hall, Englewood Cliffs, NJ.

[4] Gim, G., and Nikravesh, P., 1993. "Joint coordinate method for analysis and design of multibody systems: Part 2. system topology". KSME Journal, 7(1), pp. 26-34.

[5] Jerkovsky, W., 1978. "The structure of multibody dynamics equations". Journal of Guidance, Control, and Dynamics, 1(3), 2014/01/03, pp. 173-182.

[6] Cormen, T., 2009. Introduction to algorithms. MIT Press, Cambridge, Mass.

[7] Costello, R., Teillant, B., Weber, J., and Ringwood, J. V., 2012. "Techno-economic optimisation for wave energy converters". In 4th International Conference on Ocean Energy. 